\title{
Functional Tests Sponsor-Defined Identifier
}

National Cancer Institute

\section{Source}

National Cancer Institute. Functional Tests Sponsor-Defined Identifier. NCI Thesaurus.

Code C162132.

One or more sponsor-defined characters used to identify, name, or characterize the functional tests. 